\title{
Effects of creative dance-based exercise on gait performance in adolescents with cerebral palsy
}

\author{
Hee Joung Joung ${ }^{1}$, Jaebum Park ${ }^{1,2}$, Jooeun Ahn ${ }^{1,2}$, Moon Seok Park ${ }^{3, *}$, Yongho Lee ${ }^{1,2, *}$ \\ 'Department of Physical Education, Seoul National University, Seoul, Korea \\ ${ }^{2}$ Institute of Sport Science, Seoul National University, Seoul, Korea \\ ${ }^{3}$ Department of Orthopaedic Surgery, Seoul National University Bundang Hospital, Bundang, Korea
}

The purpose of this study is to explore the feasibility and therapeutic potential of creative dance (CD) based exercise as a rehabilitation intervention for adolescents with cerebral palsy (CP). Participants were 10 adolescents with spastic CP (mean age, $17.5 \pm 2.12$ years; Gross Motor Function Classification System levels I [ $n=3]$ and II [n=7]). Outcome measures included the Gross Motor Function Measure-88 (GMFM-88; dimensions $D$ and $E$ ), spatiotemporal gait parameters, lower limb range of motion during walking, and body image, assessed using the Body Cathexis Scale (BCS). CD was provided in 2-hr classes, twice weekly, for 12 weeks, during which participants learned movement concepts and developed their own movement. All participants completed the intervention, with an attendance rate of $98 \%$ and high satisfaction rating.
GMFM-88 dimensions $D(P=0.01)$ and $E(P=0.005)$; walking speed $(P=$ $0.005)$, cadence $(P=0.009)$, step $(P=0.005)$, and stride length $(P=0.005)$; and sagittal ranges of motions of hip $(P=0.009)$ and ankle $(P=0.03)$ during walking were significantly improved. The time of opposite foot off $(P=0.028)$ and first double-limb support $(P=0.028)$ significantly decreased, whereas the percentage of single-limb support time $(P=0.02)$ increased. Additionally, BCS scores were significantly improved. In conclusions, CD-based exercise can improve gross motor function, gait performance, and body image in adolescents with CP.

Keywords: Cerebral palsy, Creative activity, Dance-based activity, Mobility

\section{INTRODUCTION}

Cerebral palsy (CP) is a neurodevelopmental disorder that results in the abnormal development of movement and posture, often associated with sensory impairments, affecting the individual's ability to move, participate in activities, solve problems, and live independently. Although there is no evidence that damage to the brain in CP can be reversed (Ketelaar et al., 2001), a creative intervention could allow children and adolescents with CP to think about using movement differently, as well as being open to new movement ideas (Chen, 2001; Joung and Lee, 2019). Therefore, rehabilitation interventions should not only focus on functional skill development, but also on providing new opportunities and challenges that encourage individuals with $\mathrm{CP}$ to develop their maximum movement potential, as well as to achieve a positive attitude toward being challenged to explore new movement possibilities and to enjoy moving (Verschuren et al., 2012).

Recently, dance has been recognized as a promising and unique intervention in rehabilitation (Demers et al., 2015; Dhami et al., 2015). Numerous studies have demonstrated the benefits of using dance, which provides multisensory stimuli, to improve physical fitness, balance, mobility, and cognition, as well as psychological well-being, social interaction, and positive feelings in different neurological populations (Hackney et al., 2012; Heiberger et al., 2011; Marchant et al., 2010). While dancing, participants need to respond to music, verbal instructions and visual cues simulta-

\footnotetext{
*Corresponding author: Moon Seok Park (D) https://orcid.org/0000-0002-2856-7522 Seoul National University Bundang Hospital, 82 Gumi-ro 173beon-gil, Bundang-gu, Seongnam 13620, Korea

E-mail:pmsmed@gmail.com

${ }^{*}$ Co-corresponding author: Yongho Lee (iD https://orcid.org/0000-0002-6430-5327 Department of Physical Education, Seoul National University, 1 Gwanak-ro, Gwanak-gu, Seoul 08826, Korea

E-mail: gophers@snu.ac.kr

Received: May 25, 2020 / Accepted: June 20, 2020
} 
neously, experiencing movement through space (Cruz-Ferreira et al., 2015; Kattenstroth et al., 2010; Keogh et al., 2009). Various dance types have been used in a therapeutic setting, including tango (Hackney and Earhart, 2009), jazz (Alpert et al., 2009), traditional (Eyigor et al., 2009), contact improvisation (Marchant et al., 2010), and creative dance (CD) (Joung and Lee, 2019), and studies show that dance can actually improve physical function in various patient populations. Teixeira-Machado and DeSantana (2019) demonstrated that a dance intervention based on the principles of the Bartenieff, Feldenkrais and Laban methods significantly increased lower limb range of motion (ROM) in individuals with CP. Marchant et al. (2010) showed that contact improvisation dance produced significant improvements in mobility in individuals with Parkinson disease (PD).

Among the various types of dance, we specifically consider $\mathrm{CD}$ as an auspicious therapeutic intervention to facilitate movement development in children and youths with CP. Unlike structured dance forms, in which participants memorize specific movement sequences or patterns, $\mathrm{CD}$ provides ample opportunity to explore and discover unique movements based on individual functional abilities, with an infinite number of possible shapes and movements that can be explored, practiced and learned (Stribling and Christy, 2017). In CD, participants learn how to transform concepts, ideas or feelings into movement in different ways by exploring combinations of movement concepts such as body, time and space. The use of movement concepts is an effective way to encourage participants to develop their movement repertoire, to experiment with movement possibilities available to them, and to develop problem-solving abilities by creating novel solutions (i.e., new movements) (Yamaguchi and Kadone, 2017). Additionally, $\mathrm{CD}$ can allow people to creatively address their limitations, and encourages the participants to collaborate with peers in creating a dance (Joung and Lee, 2019). This feature of CD can facilitate the participation of the adolescents with $\mathrm{CP}$ in school activities and lead to the enjoyment of movement-based activities. The CD tasks in previous studies actually shared common characteristics with recent rehabilitation interventions that focus on playful practice of movement in an enriched environment, and active exploration of movement, rather than passively repeating what are considered to be normal movement patterns (Morgan et al., 2013; Morgan et al., 2015).

Despite all these potential and proved benefits, dance has not been widely used in rehabilitation (Demers et al., 2015; Dhami et al., 2015). Demers et al. (2015) pointed out that implementing dance in rehabilitation settings has been hampered by lack of skills and knowledge of the clinicians. Keogh et al. (2009) also stated that various dance programs should be developed in a professional manner to specifically address different health conditions. It is necessary to provide specific descriptions regarding a therapeutic dance intervention, and the scientific evidence of the therapeutic efficacy of that specific dance intervention. Here, we propose an actual application of a $\mathrm{CD}$ program for adolescents with $\mathrm{CP}$ based on the elements of movement, and evaluate the effect of the proposed program on gross motor function, gait performance, and body image. We hypothesized that our CD intervention would improve all these three outcomes, and verified the hypothesis with experiments.

\section{MATERIALS AND METHODS}

\section{Participants}

Thirteen participants with $\mathrm{CP}$ were recruited from Bundang Seoul National University Hospital. The inclusion criteria were as follows: (a) diagnosis of spastic CP, confirmed by an orthopedist via a clinical examination, (b) age, 13 to 20 years, (c) able to walk independently for 10 feet, without an assistive device, (d) a Gross Motor Function Classification System (GMFCS) Level I or II, and (e) able to complete the health screening questionnaire of the Bundang Seoul National University Hospital and to understand and follow the study protocol. Exclusion criteria were as follows: (a) inability to follow simple commands, (b) selective orthopedic surgery within 1 year prior to the start of the program, and (c) the use of walking aids and orthoses. We provided participants and their parents with a summary of the study protocol, and written informed consent was obtained from the participants and their parents before enrollment and data collection. The study protocol was approved by the Clinical Research Ethical Committee of Bundang Seoul National University Hospital (B-1707-408-302).

\section{Study design}

This was a single-group cohort study, with pre- and postintervention comparisons. All assessments took place in the Motion Analysis Laboratory at Bundang Seoul National University Hospital. Participants completed a three-dimensional gait analysis and the Gross Motor Function Measure-88 (GMFM-88) and body image questionnaires 7 days before and 7 days after the intervention. Additionally, participants completed a satisfaction questionnaire 1 week after the intervention. All assessments were evaluated by the same examiner. Participants completed our 12-week CD program, offered in 2-hr session, twice per week, for a total of 


\begin{tabular}{|llll|}
\hline & \multicolumn{3}{c|}{ Concepts } \\
\hline Body & C1 & Body & upper, lower body parts \\
\hline Time & C2 & Speed & fast - slow \\
\hline Space & C3 & Level & high - low \\
\cline { 2 - 4 } & C4 & Size & big - small \\
\cline { 2 - 4 } & C5 & Shape & number, name, etc. \\
\cline { 2 - 4 } & C6 & Direction & (b) \\
\cline { 2 - 4 } & C7 & Pathway & (c) \\
\hline
\end{tabular}
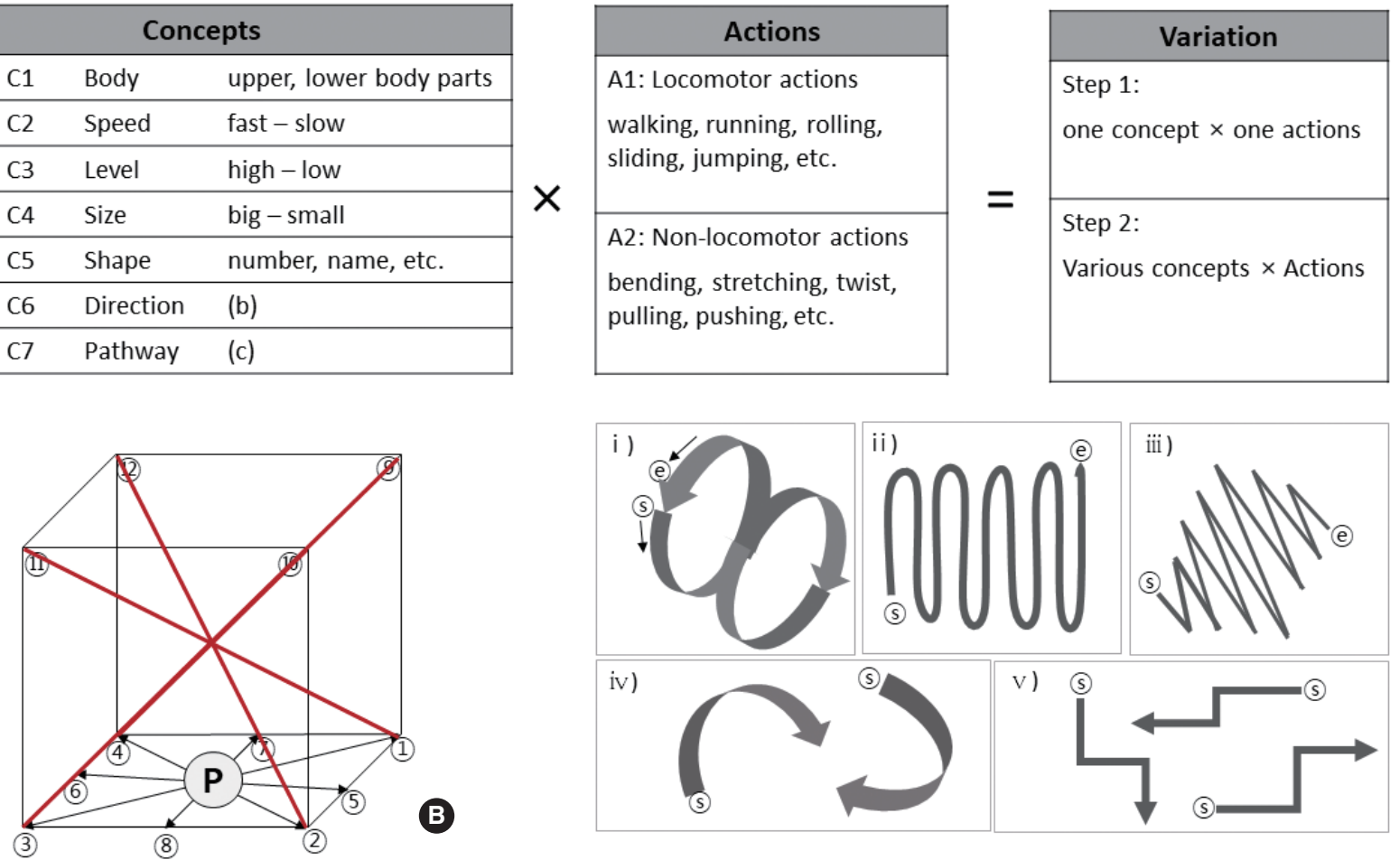

Fig. 1. The conceptual framework of the Lets be Creative Dance program to increase the repertoire of movement. (A) The movement variation principle. (B) Visual material for direction elements: $P=$ standing positions; (1)-(4) straight paths, (5)-8) addition of diagonal paths, and (9)-(12) reaching with the arms toward different spatial squares. The red line is a virtual line for reaching movements of the arms and legs away from the center of mass. (C) Paths used: (i) Fig. 8-shaped path, (ii) U-shaped paths, (iii) a zigzag path, (iv) a half-circle path, and (v) a perpendicular line.

24 sessions. We developed the conceptual framework for movement variation and a $\mathrm{CD}$ protocol for our Lets be Creative Dance Program for Movement Variation. A professional contemporary dance choreographer led the $\mathrm{CD}$ sessions.

\section{The CD-based exercise}

\section{Conceptual framework of the Let's Be Creative Dance Exercise program}

The conceptual framework of our CD program was based on the movement concept (Joung and Lee, 2019) (Fig. 1). The goal of this framework was for the participants to develop their own movements and to explore ways of incorporating movement through the learning of movement concepts.

\section{The Let's Be Creative Dance Exercise program}

All CD tasks reflected this principle and encouraged participants to actively explore their environment through trial and error. The $\mathrm{CD}$ tasks were scaffolded, providing multisensory stimulation to achieve the goal of diversification of voluntary movement. All CD tasks attempted to accomplish the overall goals of the program by providing opportunities to explore and experiment with a variety of movement possibilities though improvisation, rather than through repetition of the same actions. Improvisation is an effective method to begin to develop one's own movement in the CD process (Moffet et al., 2000; Stribling and Christy, 2017). This program is divided into three stages to help people learn to move step by step and to motivate interest for performing creative movement: the play, move and dance stages (Joung and Lee, 2019).

The purpose of the play stage (Table 1) is to obtain the idea of movement through games. The use of games in the play stage is also important to help participants mitigated any fear they may have of creating their own movements and showing their movements to others. Tasks in the play stage focused on body and spatial awareness. In the play stage, participants learned how to combine one action with one concept. Understanding the movement concepts provided concrete direction so that the participants who were new to $\mathrm{CD}$ more clearly understood how to create their movement and, thus, learn to apply a movement concept to actions. Though this exploration, participants learned how actions could 
Table 1. Description of the tasks in the play stage

\section{Task description}

\section{Task 1. Drawing shapes with body parts}

- Participants drew shapes (e.g., circle, straight, name, signature) in the air with body parts, starting with an isolated body part (e.g., head as a single point), and then adding one or two more body parts at a time (e.g., head×shoulder, shoulder $\times$ pelvic $\times$ knee). "Let's draw a circle by moving the head and shoulder together."

-While drawing, an instructor randomly called out different elements of time (slow to fast), level (low to high), shape, place, or body part. By changing these elements, participants explored a variety of ways to move the body parts, encouraging the use of different movement elements (level, speed, direction, etc.).

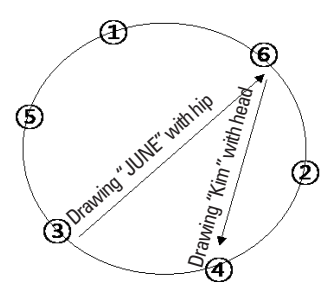

Task 2. Human rock-paper-scissors with body parts

- Participants made 'rock-paper-scissors' actions while exploring the elements of shape and size. Participants walked in a scattered formation, and then, after a stop signal, performed one of the rock-paper-scissor actions with another participant who was met by chance. (a) Rock=contraction; becoming small by bending down, hugging the knees, and curling into a ball. (b) Paper= expansion; becoming big by stretching the arms and legs using the biggest movements possible. (c) Scissors=sharp; squeezing the legs and balancing on one leg as much as possible.
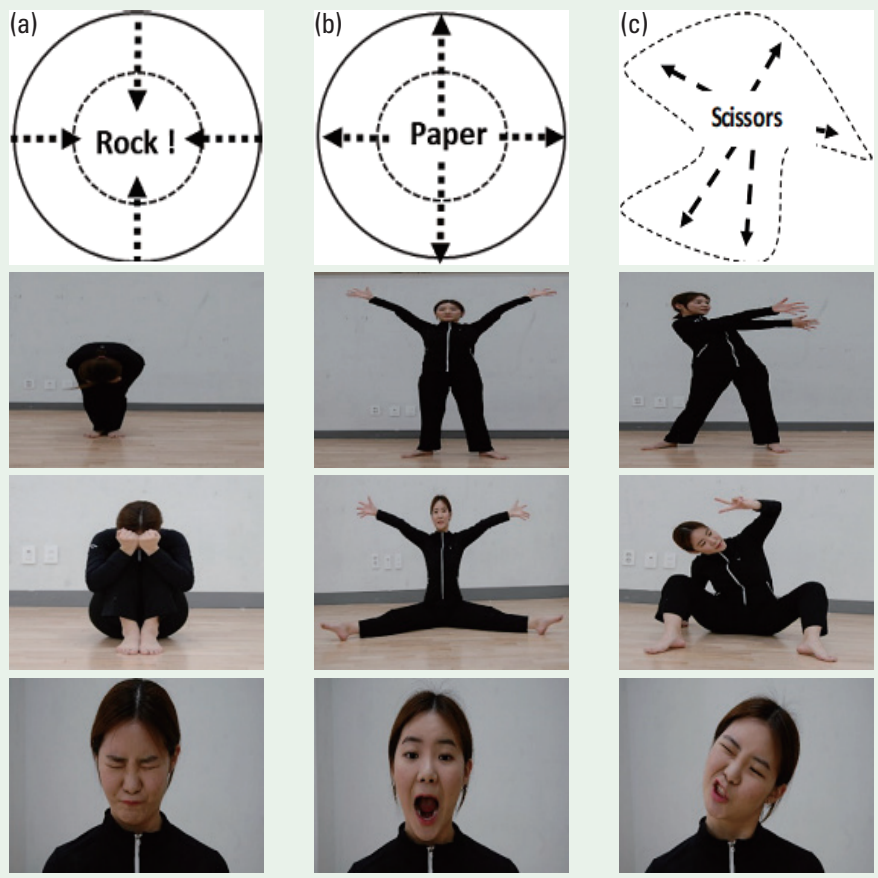

Task 3. Model walking task

- Participants practiced locomotor skills and improved their self-confidence in dancing in front of others. We implemented the following strategy to help participants overcome their shyness and/or embarrassment of dancing freely in front of people. Three participants, as models, performed locomotor actions (e.g., walk, run, or roll) and poses at three different levels (high-middle-low). The other participants, as the audience, watched and cheered.

3 poses at a high-middle-low level

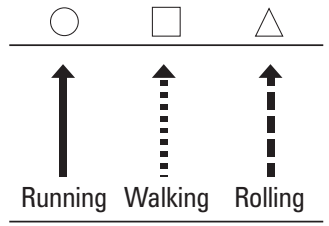

"Sit audience"

\section{Task 4. Mirroring task}

- Participants copied a partner's movements. Two participants faced each other. One was the leader and the other was the follower. When the leader moved, the follower copied their movements as closely as possible. Several people could be followers.

- The leader was asked to move as slowly as possible in order to allow the follower to copy. After a signal, the participants switched roles.

- This task allowed participants to expand their range of movement by experiencing another's movement and improved interaction among participants.

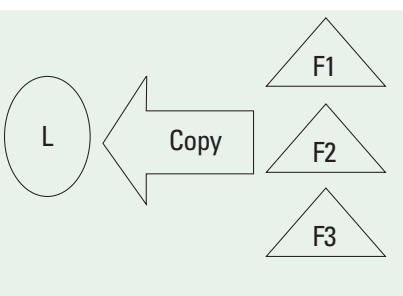

(Continued to the next page) 
Table 1. Continued

\section{Task description}

\section{Task 5. Human domino}

- Starting in a circle formation ( $i=$ starting position, $\rightarrow$ =moving direction), each participant successively delivered a ball to the participant next to them (a). Participants then randomly delivered the ball across the inner circle (b).

- An element of time could be added (e.g., clap domino while slowly walking or drawing name with one body part) and several starting points can be used (c and d). Furthermore, the domino task can be performed with actions other than delivering a ball (e.g., clap domino, push with elbow domino, and drawing name domino).
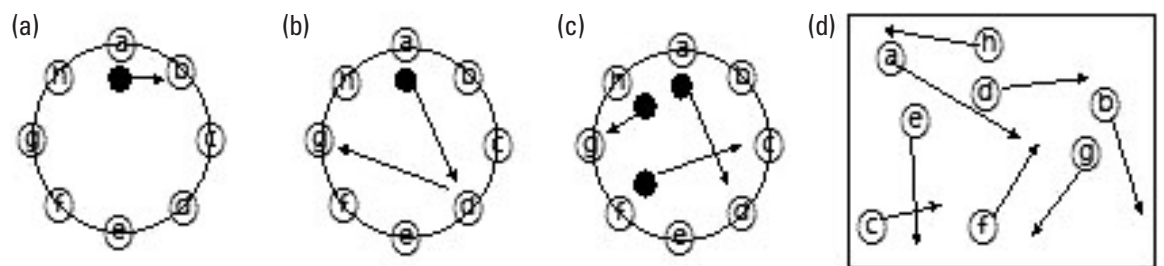

Task 6. Random walking

- Task 5 (the domino task) can be devolved into task 6 if participants are not familiar with walking in various directions (see visual description task 5 d). Task 6 aims to develop the skills necessary to walk in various directions.

- Participants walked using their own choice of movement concepts (e.g., direction, times, pathways, shapes) in a scattered formation and pointed (with their fingers) the direction of their movement to help them become more aware of their direction.

- Initially, participants walked around the classroom in a scattered formation. While walking, the instructor randomly called out different directions (e.g., walking forward, backward, or sideways), speeds (slowly to quickly), pathways (e.g., walk in a straight, curved, or zigzag path), shapes (e.g., walk in the shape of a circle, a letter of the family name, or a number, such as a big or small ' 8 '), resulting in walking $\times$ pathway $\times$ time $\times$ size $\times$ shape combinations.

- The instructor encouraged participants to spontaneously apply movement concepts to walking. Participants voluntarily tried to walk in various directions, through several pathways, with controlled walking speed, or make various shapes with their body parts, thereby developing spatial awareness. While walking, participants might meet by chance; thus, they were asked to avoid shoulder-to-shoulder situations and keep a distance from others. The instructor encouraged participants to make eye contact with members, or high-five each other, and to look for an empty space in which to move through.
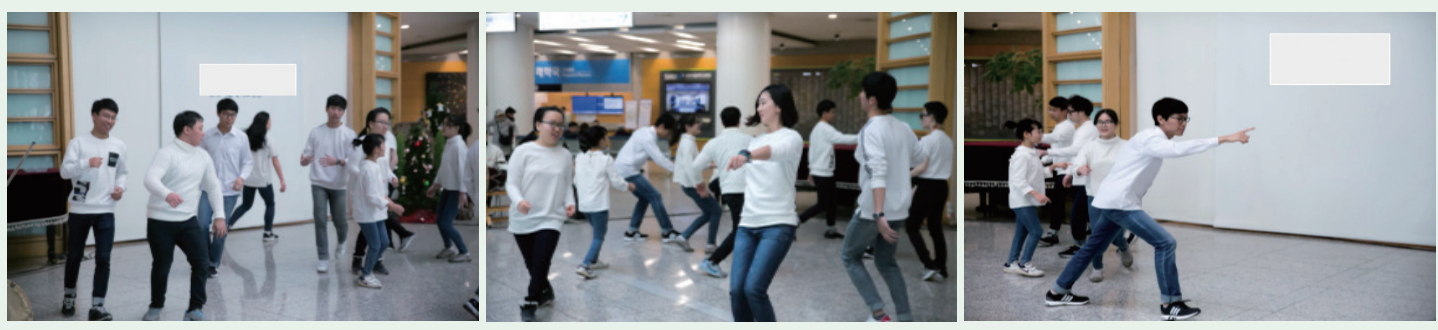

All photos were reproduced with permission; participants provided written informed consent concerning the use of the photographs.

be changed depending on the level of each movement concept.

The purpose of the move stage (Table 2) was to develop sequential movement patterns by improving skills in combining dance concepts. Tasks focused on exploring ways of incorporating movement by responding to sensorimotor stimuli (e.g., auditory, visual and tactile cues) under conditions of multitasking. These tasks helped participants to develop a wider range of movement possibilities to draw upon for expression. In the move and dance stage, participants learned how to combine one of the actions with two or more concepts by developing movement diversification. There was no limit to the possibility of movement variation that could be explored, depending on the combination of concepts, as well as to experiment with a greater variety of movement possibilities.

The purpose of the dance stage was to adapt new movement patterns to create a dance piece using movement skills learned during the previous play and move stages. With a focus on creation, participants discussed their interests in daily life in order to select a dance topic related to 'meeting on the street.' This dance piece was performed at the Bundang Seoul National University Hospital, and the total running time of the dance performance was 30 $\min$.

\section{Outcome measures}

\section{Spatiotemporal gait parameters and sagittal hip, knee, and ankle ROM}

Kinematic data during walking were collected using a 10-camera Vicon Motion Analysis System (Motion Analysis Corp., Rohnert Park, CA, USA), at a sampling frequency of $120 \mathrm{~Hz}$. The Helen 
Table 2. Description of the tasks in the movement stage

Task description

\section{Task 7. Improvisation on a theme}

- Topics included activities of daily life at home (e.g., actions in the bedroom, living room, bathroom, and kitchen) and in the environment (e.g., meeting someone, picking up a coin, and waiting for a bus). Participants travelled around the classroom in a scattered formation. When the music stopped, participants performed an action. This was repeated several times to target the various tasks. The participants were then encouraged to apply concepts (speed, level, size, and direction) to their actions, such as very slowly shaking their body, performing big whole-body movements, or using different body parts during the waiting for a bus action.

Task 8. Dance by chance

- This task encouraged participants to quickly respond to auditory stimuli and change actions.

- Participants were divided into several small groups. (a) Each group selected three actions (e.g., $1=$ reaching arms, $2=$ jumping, and $3=$ rolling). The instructor then randomly called out numbers and participants performed each corresponding action. (b) Two groups simultaneously performed their selected actions following the instructor's directions.

(a)

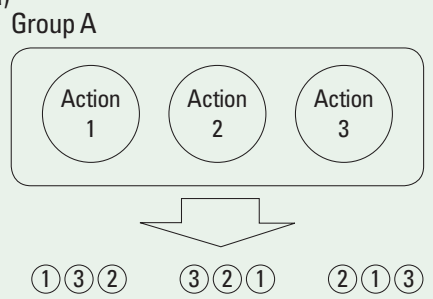

(b)

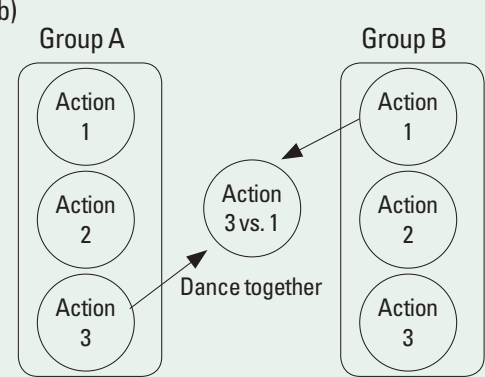

Task 9. Pathway task: see Fig. 1C

- This task allowed participants to practice walking and running along different paths.

- Pathways included Fig. 8-shaped and U-shaped curved paths, circular paths, zigzags, and perpendicular paths. Participants were visually shown these paths to enhance their understanding. Walking along curved, circular, and zigzag paths requires sudden weight shifting, changes in the position of the center of gravity, and different rotations of the torso.

Task 10. Hand airplane

- This task focused on improvising movement patterns using the hands, with the hands playing the role of an airplane.

(a) Single-person hand airplane. Initially, participants used one hand as an airplane. The hand airplane flew into the air, drawing various pathways. One hand drives the movement and the torso moved to follow the direction of the hand. The hand plane then landed slowly on the floor, with placing of a hand on the floor mimicking the landing of the plane at the airport. The instructor suggested the destination (e.g., Seoul to New York, or Korea to Africa), which indicated how long the participants should continue their movement. Later, both hands became airplanes. The right hand flew and stopped in the air. Then the left hand flew, with a landing on the right hand, which was a sign for the right hand to move. Participants could experiment with many different movement patterns in order to find solutions to new demands.

(b) Hand airplane with a partner. One hand airplane landed on a partner's body part while the partner did not move at all (like a statue). The landing of the hand was the sign to move; the partner then flew and landed a hand airplane on the other participant's body part, continuing the cycle of interaction.

(a)

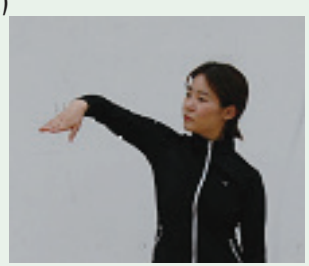

(b)

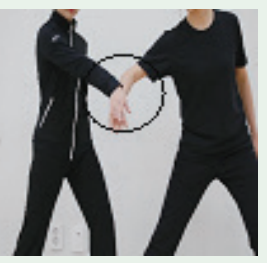

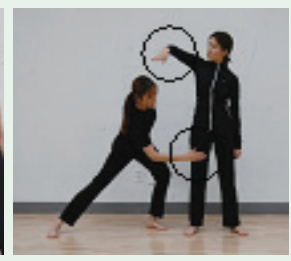

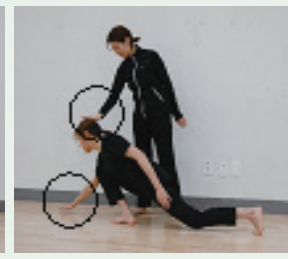

(c)
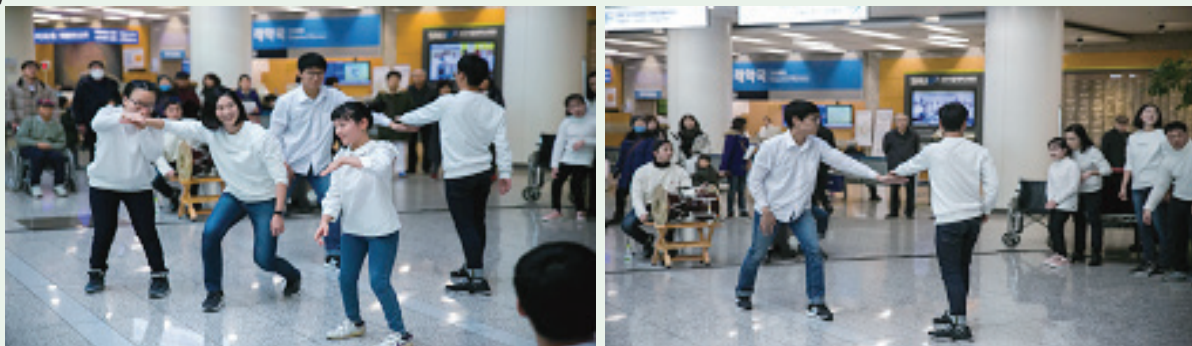

Using hand air plane in dance performance

All photos were reproduced with permission; participants provided written informed consent concerning the use of the photographs. 
Hayes 16-marker arrangement was used, with markers attached, bilaterally, to the anterior superior iliac spine, sacrum, anterior aspect of the mid-thigh, lateral femoral epicondyle of the knee, lateral aspect of the mid-calf, lateral malleolus, base of the heel, and dorsum of the foot, between the second and third metatarsals. Participants walked barefoot along a 9-m walkway, with more than three trials completed for kinematic data recording. Data for the three best trials (representing each participant's typical gait pattern) were selected and averaged. Spatiotemporal parameters, including walking speed, cadence, step and stride length, and single- and double-limb support time, were quantified. The ROMs of the pelvis and of the knee and ankle joints, in the sagittal plane, were additionally calculated.

\section{Gross Motor Function measurement}

The GMFM-88 (Avery et al., 2003; Ko and Kim, 2013) is a standardized instrument used to measure change in the gross motor function of children and youths with $\mathrm{CP}$ and has been used assess outcomes of an intervention among children and youths with CP. The GMFM consists of 88 items, distributed across the following five dimensions: (A) lying and rolling; (B) sitting; (C) crawling and kneeling; (D) standing; and (E) walking, running, and jumping. We evaluated the $\mathrm{D}$ and $\mathrm{E}$ dimensions in this study. A percentage score was calculated for each dimension, using the Gross Motor Ability Estimator. The same examiner evaluated the GMFM- 88 before and after the intervention.

\section{Body image}

Body image was assessed using the Body Cathexis Scale (BCS), which was developed by Secord and Jourard (Jourard and Secord, 1955). The BCS score is calculated on a 5-point Likert scale, with responses ranging from "I don't like it at all and I wish it could be changed." to "I am completely satisfied and I would not change it if I could." Split-half reliabilities of the BCS score are high, ranging from 0.88 to 0.92 (Jourard and Secord, 1955).

\section{Statistical analysis}

All data were analyzed using IBM SPSS Statistics ver. 21.0 (IBM Co., Armonk, NY, USA). The statistical significance level was set at $<0.05$. Data were expressed as a mean \pm standard deviation. Descriptive statistics were calculated for all variables. The Shapiro-Wilk test was used to evaluate the normality of data distribution. Nonparametric tests, using the Wilcoxon singled-rank test for pairwise comparisons, were performed to evaluate pre- and postintervention changes in the GMFM-88 score and spatiotem-
Table 3. General characteristics of the participants

\begin{tabular}{clcccc}
\hline No. & Sex & Age (yr) & Height $(\mathrm{cm})$ & Weight (kg) & GMFCS (level) \\
\hline 1 & Female & 13 & 138.2 & 33 & $\|$ \\
2 & Female & 15 & 150.5 & 48.7 & $\|$ \\
3 & Male & 17 & 163.6 & 51 & I \\
4 & Female & 17 & 156.8 & 63.4 & $\|$ \\
5 & Male & 18 & 162 & 75.3 & $\|$ \\
6 & Male & 18 & 160 & 46.3 & I \\
7 & Male & 19 & 162 & 65 & $\|$ \\
8 & Male & 19 & 164.4 & 64.5 & I \\
9 & Male & 19 & 158 & 61.8 & $\|$ \\
10 & Male & 20 & 162 & 31.2 & $\|$ \\
\hline
\end{tabular}

GMFCS, Gross Motor Function Classification System.

poral gait parameters. A paired $t$-test was used to evaluate differences pre- and postintervention changes in sagittal plane hip, knee and ankle ROM.

\section{RESULTS}

\section{General characteristics of the participants}

Thirteen participants were eligible for enrollment. Three participants dropped out due to medical issues prior to the start of the program. Therefore, our analysis was based on the data of 10 participants with spastic CP (GMFCS level I, $\mathrm{n}=3$, and level II, $\mathrm{n}=7$; age, $18.5 \pm 3.37$ years; height, $157.8 \pm 7.98 \mathrm{~cm}$; and weight, $57.02 \pm 12.17 \mathrm{~kg}$ ). Relevant details of these 10 participants are provided in Table 3.

\section{Change in the spatiotemporal gait parameters}

Changes in the spatiotemporal gait parameters after the $\mathrm{CD}$ intervention, relative to preintervention values, are presented in Table 4 . The percentage of opposite foot off change $(P=0.028$, $\Delta-12.31 \%)$ and the first double-limb support time $(P=0.028$, $\Delta-12.31 \%)$ significantly decreased after the intervention, while single-limb support time $(P=0.022, \Delta+5.03 \%)$ increased. Additionally, step length $(P=0.005, \Delta+7.66 \%)$, stride length $(P=$ $0.005, \Delta+8.35 \%)$, cadence $(P=0.009, \Delta+5.40 \%)$, and walking speed $(P=0.005, \Delta+13.41 \%)$ all increased after the intervention. There was no significant change in foot off time and in the second double-limb support time.

\section{Change in sagittal plane hip, knee, and ankle ROM over the gait cycle}

Changes in sagittal plane hip, knee and ankle ROM over the gait cycle after the $\mathrm{CD}$ intervention, relative to preintervention 
Table 4. Changes in spatiotemporal gait parameters

\begin{tabular}{lccrc}
\hline Variable & Preintervention & Postintervention & Change (\%) & $P$-value \\
\hline Opposite foot off (\%) & $12.83 \pm 3.12$ & $11.25 \pm 3.35$ & 12.31 & 0.028 \\
Opposite foot strike (\%) & $49.84 \pm 0.54$ & $50.13 \pm 0.51$ & 0.58 & 0.240 \\
Foot off (\%) & $62.73 \pm 3.07$ & $61.25 \pm 3.55$ & 2.36 & 0.059 \\
$\begin{array}{l}\text { First double-limb } \\
\quad 12.83 \pm 3.12\end{array}$ & $11.25 \pm 3.35$ & 12.31 & 0.028 \\
$\quad$ support (\%) & & & & \\
Second double-limb & $13.02 \pm 3.44$ & $11.12 \pm 3.61$ & 14.59 & 0.590 \\
$\quad$ support (\%) & & & & \\
Single-limb support (\%) & $37.0 \pm 3.30$ & $38.86 \pm 3.36$ & 5.03 & 0.022 \\
Step length (cm) & $47.64 \pm 6.37$ & $51.29 \pm 5.95$ & 7.66 & 0.005 \\
Stride length (cm) & $94.69 \pm 12.25$ & $102.6 \pm 11.58$ & 8.35 & 0.005 \\
Cadence (steps/min) & $114.7 \pm 12.11$ & $120.9 \pm 8.84$ & 5.40 & 0.009 \\
Walking speed (cm/s) & $90.73 \pm 13.08$ & $102.9 \pm 11.36$ & 13.41 & 0.005 \\
\hline
\end{tabular}

Values are reported as the mean \pm standard error.

$P$-values are calculated from paired t-tests, comparing postintervention with preintervention values. values, are presented in Fig. 2A, B. The intervention yielded an increase in right hip $(P=0.009, \Delta+9.91 \%)$ and ankle $(P=0.003$, $\Delta+15.87 \%)$ ROM.

\section{Change in the Gross Motor Function Measure Score}

Changes in the score on dimensions $\mathrm{D}$ (standing) and $\mathrm{E}$ (walking, running, jumping) of the GMFM- 88 after the CD intervention, relative to preintervention values, are presented in Fig. 2C. Significant improvements in both dimensions were identified: $\mathrm{D}$, $P=0.011, \Delta 10.53 \%$; and $\mathrm{E}, P=0.005, \Delta+17.29 \%$.

\section{Change in the BCS Score}

Changes in the BCS score after the $\mathrm{CD}$ intervention, relative to preintervention values, are shown in Fig. 2D. The BCS score increased after the intervention $(P=0.005, \Delta 25.96 \%)$.
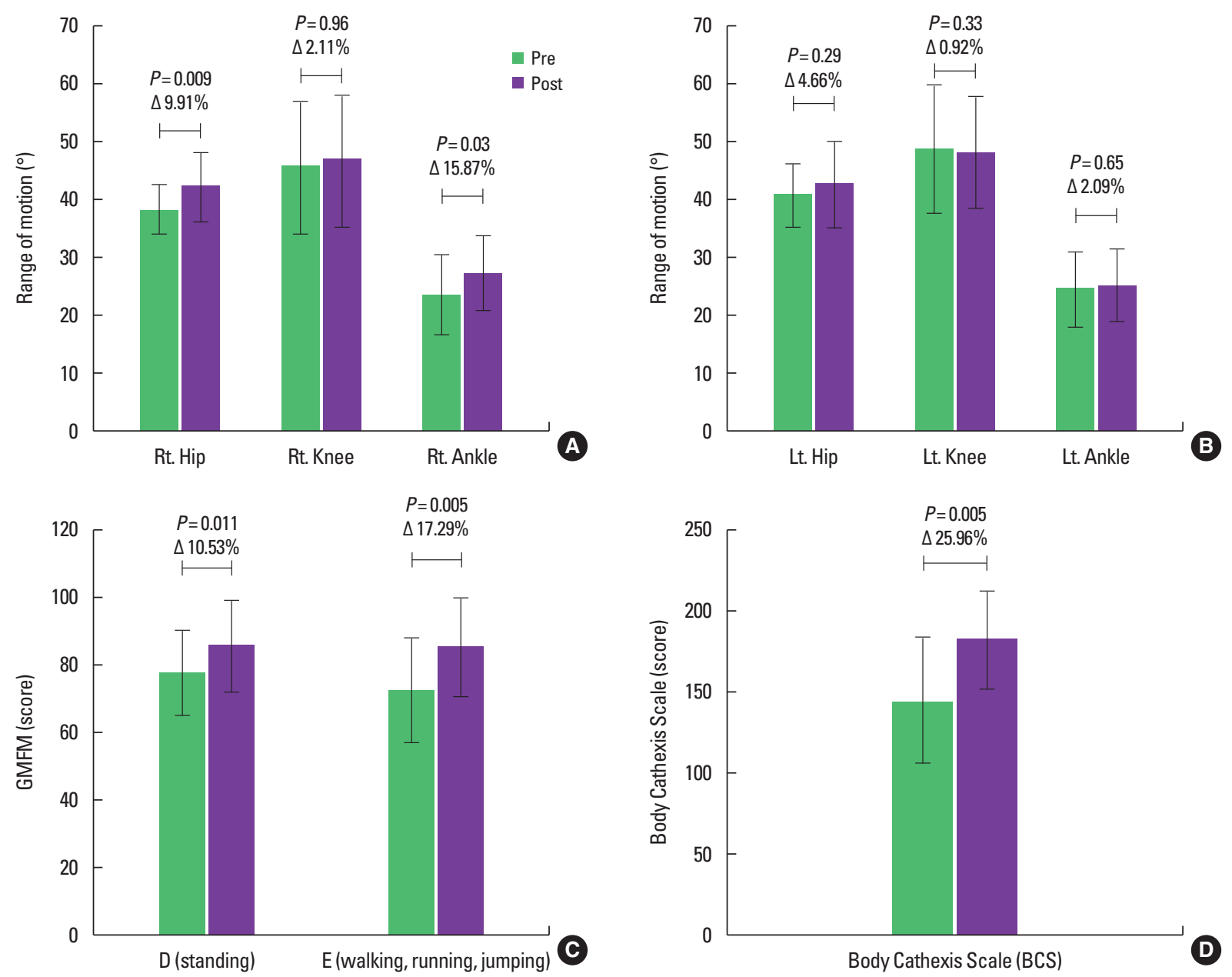

Fig. 2. Changes in the sagittal plan range of motion of the right hip, right knee, right ankle (A) and left hip, left knee, left ankle (B) over the gait cycle; (C) the Gross Motor Function Measurement (GMFM)-88 score; (D) the Body Cathexis Scale score. Values are reported as the mean \pm standard error. Comparison of pre- to postintervention changes were evaluated using paired $t$-tests. 


\section{DISCUSSION}

The purpose of our study was to explore the feasibility of CDbased exercise as a potentially beneficial rehabilitation intervention to improve movement and mobility among adolescents with CP. Twelve weeks of the CD resulted in the following: (a) a decrease in the time of opposite foot off and first double-limb support time; an increase in single-limb support time, walking speed, stride length, step length, and cadence; (b) an increase in right hip and ankle sagittal ROM over the gait cycle; (c) an increase in the GMFM-88 score on dimension D (standing) and $\mathrm{E}$ (walking, running, and jumping); and (d) an improvement in body image. All participants showed excellent attendance (98\%) and a high level of satisfaction with the program.

The observed improvements in sagittal plane hip and ankle ROM may explain the improvement in spatiotemporal gait parameters previously reported with a dance intervention (De Bujanda et al., 2003). Moreover, improvement in hip extension and flexion ROM contributes to increasing stride length and walking speed (Ardestani et al., 2016). In addition, Moffet et al. (2000) reported that 8 weeks of $\mathrm{CD}$-based exercises resulted in improvements in spatiotemporal gait parameters (including walking speed, cadence, stride length, and cycle duration) and kinematic variables (including total hip range and maximum flexion during stance) among patients with rheumatoid arthritis (functional class III; age, $54 \pm 9$ years). Teixeira-Machado and DeSantana (2019) demonstrated that 12 weeks of a creative movement program, with two sessions per week, improved the passive ROM, in all axes of motion, of the hip, knee and ankle joints among young people with CP (GMFCS level III-V; age, 13-14 years) to a greater extent than a program of neurodevelopmental treatment or proprioceptive neurodevelopmental treatment. Marchant et al. (2010) showed that 2 weeks of contact improvisation dance improved the relative duration of the stance and swing phases of the gait cycle, as well as the step length, during backward walking in people with PD. Finally, weakness of the hip abductor muscles is related to an unstable gait pattern and less time spent in single-limb support in children with spastic diplegic CP (Gage and Novacheck, 2001). Thus, our findings of a significant increase in single-limb support time, accompanied by significant increases in stride and step length and walking speed after the $\mathrm{CD}$ intervention, are consistent with previous studies that support a relationship between improvements in ROM and gait performance.

Our $\mathrm{CD}$ program also yielded improvements in the dimension $\mathrm{D}$ and $\mathrm{E}$ scores of the GMFM-88 scores. A previous study report- ed a high correlation between the dimension E score and walking speed, while the score on dimension $\mathrm{D}$ (standing) reflected the elementary function of gait (Drouin et al., 1996). Thus, our findings further support the utility of the GMFM-88 dimension E score as a predictor of locomotor performance, and provide evidence of the positive impact of $\mathrm{CD}$ tasks on gross motor function and gait performance in adolescents with $\mathrm{CP}$.

Although our results demonstrate the positive effects of $\mathrm{CD}$ on movement and gait capacity among adolescents with $\mathrm{CP}$, the lack of scientific evidence regarding the use of $\mathrm{CD}$ with children with $\mathrm{CP}$ restricts our discussion. Additionally, our own study is limited by the small sample size and nonrandomized study design. Potential sources of bias, such as confounding factors, and a selection bias may have affected the observed improvements in gait performance and gross motor function. This work is the lack of a control group receiving a different intervention, which is inherent in an exploratory feasibility study. In addition, as the present study was a pilot study, we did not include a follow-up phase to determine how long the improvement lasted after the intervention. Thus, the results of this small feasibility study need to be interpreted with caution. We can, however, summarize the important features of a $\mathrm{CD}$ program based on the results of the present study, in order to provide more concrete information to practitioners in terms of implementing a $\mathrm{CD}$ intervention in rehabilitation (Demers et al., 2015).

Next, we have described features of $\mathrm{CD}$ based on the results of the present study, as it may provide more detailed information on why gross motor skills, gait performance, and body image were improved. First, the body awareness tasks (tasks 1, 2, 3, 4, 7, 9, and 10) focused on whole-body connectivity. These tasks encouraged participants to explore the dynamics of their actions with a single part of body as a first step (unitary sequencing), and then gradually connected two or more parts of the body (simultaneous sequencing). For example, in task 1 (drawing a shape with body parts), participants moved a body part (e.g., head, neck, shoulder, arms, hip, or leg) while drawing a shape (e.g., circle, name). In body-connection tasks, participants moved two body parts simultaneously, responding to random verbal cues (e.g., shoulder and hip, arms and knee). After this work, the teacher progressively added speed (e.g., fast-slow) and level (e.g., high-medium-low) elements, and participants gradually developed the ability to combine each body part into sequential and combination movements. As a result, a variety of unintended or novel movement patterns emerged and participants experienced movements of some body parts that they did not usually use in daily life or used inadequate- 
ly. This allowed participants to extend their movement possibilities, increasing the variability in their actions. These features may have contributed to the improvements in hip and ankle sagittal ROMs and stride length that we observed.

Second, the tasks for spatial awareness (tasks 5, 6, 7, 8, 9, and 10) encouraged participants to adapt their movement to dynamic conditions, responding to cues for different directions, levels and speeds. Participants practiced moving or reaching out with fingers, toes, or the head toward numbers on the cube, while pointing in the same direction. Additionally, participants practiced walking while considering unexpected stimuli (e.g., different music tempos, people, different shapes), which were randomly called out by the teachers, as well as the random and hand airplane tasks. During these tasks, participants might meet by chance, which required them to avoid shoulder-to-shoulder contact and maintain a safe distance from others, while responding to randomized multiple external stimuli. These tasks emphasized multidirectional weight-shifting while changing direction or body shapes and responding to unexpected stimuli. This is an important issue in the learning and generalization of a motor activity, as the context is similar to that in real walking situations, such as walking outdoors, on uneven surfaces, in crowded places, and across complex places (Valvano, 2004). Thus, interventions with dynamic conditions may evoke the process of adaptation in the postural control system (Granacher et al., 2011). Furthermore, the CD tasks in the present study were accompanied by music, with different tempos, rhythms, and beats. Participants changed the movement speed in response to different tempos, as suggested by the verbal cues (e.g., move slow, fast, gradually slow, or gradually fast) or music, while creating sequential movement patterns. In rhythmic auditory stimulation and music-based interventions, like Jaques-Dalcroze's eurhythmics, walking training or exercises are performed to the rhythm of improvised music, with positive effects in bringing older adults to engage in various multidirectional movement patterns related to gait performance and dynamic balance (Kressig et al., 2005).

Finally, participants reported an improvement in their body image, as assessed by the BCS. People with physical disabilities are likely to experience a negative body image (because of the physical features of their disability) and to have difficulty in establishing a positive body image (Shpigelman and HaGani, 2019). However, Taleporos and McCabe (2002) demonstrated that some aspects of positive feedback from significant others and personal achievements, such as those in sports, a career or education, can contribute to the development of a positive body image in people with physical disabilities. Participants further indicated that the repeated successful experience of their movement motivated them to be more active, and improved their confidence in their movement abilities and life situation. The participants collaborated with peers, while sharing their ideas or making a group dance sequence, and participated in a dance performance at Bundang Hospital. Compared to other activities, this is an experience unique to dance. Participants reported that they felt enthusiastic during the dance performance, when receiving applause from the audience and presenting their achievements. Importantly, the willingness to move independently and face the challenge of new events emerged in everyday life (e.g., using public transportation alone, standing up and singing in the school choir, practicing dance alone at home, joining a school dance club, and conducting a theater play) with more confidence. Thus, the positive experience and achievements in our Let's Be Creative CD program, as noted above, may have contributed to the improvement in body image.

In conclusion, the $\mathrm{CD}$ protocol and tasks that we describe in our study reflect a process of learning of how to create novel movements, develop one's own movement in order to implement self-directed activity, and to adapt motor learning processes based on one's individual strengths and learning challenges (Valvano, 2004), including improving problem-solving abilities, maximizing one's movement potential and creating novel movement patterns (Verschuren et al., 2012). As a practical methodology, it is important to include the following elements in a program of dance: engage children in the process of the creation by learning the elements of movement, which can be facilitated using our $\mathrm{CD}$ protocol; provide sufficient time and opportunity for movement exploration; include various multisensory stimuli for movement variation; and respect any movement from participants, providing positive feedback. The present study demonstrated the feasibility and potential of $\mathrm{CD}$ for adolescents with $\mathrm{CP}$. Therefore, we suggest CD-based exercise as a new form of rehabilitation intervention for children with CP.

\section{CONFLICT OF INTEREST}

No potential conflict of interest relevant to this article was reported.

\section{ACKNOWLEDGMENTS}

We gratefully acknowledge the contribution of all participants and their families. We would also like to acknowledge Bundang 
Hospital for providing technical support, and Soo Kyung Kim and Young Sin Kim for their assistance with this study.

This study was funded by the Bundang Seoul National University Hospital in Republic of Korea (02-2017-056). The study sponsors played no role in the study design, data collection, interpretation, or decision to submit for publication.

\section{REFERENCES}

Alpert PT, Miller SK, Wallmann H, Havey R, Cross C, Chevalia T, Gillis $\mathrm{CB}$, Kodandapari K. The effect of modified jazz dance on balance, cognition, and mood in older adults. J Am Acad Nurse Pract 2009;21: 108-115.

Ardestani MM, Ferrigno C, Moazen M, Wimmer MA. From normal to fast walking: impact of cadence and stride length on lower extremity joint moments. Gait Posture 2016;46:118-125.

Avery LM, Russell DJ, Raina PS, Walter SD, Rosenbaum PL. Rasch analysis of the Gross Motor Function Measure: validating the assumptions of the Rasch model to create an interval-level measure. Arch Phys Med Rehabil 2003;84:697-705.

Chen W. Description of an expert teacher's constructivist-oriented teaching: engaging students' critical thinking in learning creative dance. Res Q Exerc Sport 2001;72:366-375.

Cruz-Ferreira A, Marmeleira J, Formigo A, Gomes D, Fernandes J. Creative dance improves physical fitness and life satisfaction in older women. Res Aging 2015;37:837-855.

De Bujanda E, Nadeau S, Bourbonnais D, Dickstein R. Associations between lower limb impairments, locomotor capacities and kinematic variables in the frontal plane during walking in adults with chronic stroke. J Rehabil Med 2003;35:259-264.

Demers M, Thomas A, Wittich W, McKinley P. Implementing a novel dance intervention in rehabilitation: perceived barriers and facilitators. Disabil Rehabil 2015;37:1066-1072.

Dhami P, Moreno S, DeSouza JF. New framework for rehabilitation - fusion of cognitive and physical rehabilitation: the hope for dancing. Front Psychol 2015;5:1478.

Drouin LM, Malouin F, Richards CL, Marcoux S. Correlation between the gross motor function measure scores and gait spatiotemporal measures in children with neurological impairments. Dev Med Child Neurol 1996;38:1007-1019.

Eyigor S, Karapolat H, Durmaz B, Ibisoglu U, Cakir S. A randomized controlled trial of Turkish folklore dance on the physical performance, balance, depression and quality of life in older women. Arch Gerontol Geriatr 2009;48:84-88.

Gage JR, Novacheck TF. An update on the treatment of gait problems in cerebral palsy. J Pediatr Orthop B 2001;10:265-274

Granacher U, Muehlbauer T, Zahner L, Gollhofer A, Kressig RW. Comparison of traditional and recent approaches in the promotion of balance and strength in older adults. Sports Med 2011;41:377-400.

Hackney ME, Earhart GM. Effects of dance on movement control in Parkinson's disease: a comparison of Argentine tango and American ballroom. J Rehabil Med 2009;41:475-481.

Hackney ME, Hall CD, Echt KV, Wolf SL. Application of adapted tango as therapeutic intervention for patients with chronic stroke. J Geriatr Phys Ther 2012;35:206-217.

Heiberger L, Maurer C, Amtage F, Mendez-Balbuena I, Schulte-Mönting J, Hepp-Reymond MC, Kristeva R. Impact of a weekly dance class on the functional mobility and on the quality of life of individuals with Parkinson's disease. Front Aging Neurosci 2011;3:14.

Joung HJ, Lee Y. Effect of creative dance on fitness, functional balance, and mobility control in the elderly. Gerontology 2019;65:537-546.

Jourard SM, Secord PF. Body-cathexis and personality. Br J Psychol 1955; 46:130-138.

Kattenstroth JC, Kolankowska I, Kalisch T, Dinse HR. Superior sensory, motor, and cognitive performance in elderly individuals with multiyear dancing activities. Front Aging Neurosci 2010;2:31.

Keogh JW, Kilding A, Pidgeon P, Ashley L, Gillis D. Physical benefits of dancing for healthy older adults: a review. J Aging Phys Act 2009;17: 479-500.

Ketelaar M, Vermeer A, Hart H, van Petegem-van Beek E, Helders PJ. Effects of a functional therapy program on motor abilities of children with cerebral palsy. Phys Ther 2001;81:1534-1545.

Ko J, Kim M. Reliability and responsiveness of the gross motor function measure-88 in children with cerebral palsy. Phys Ther 2013;93:393-400.

Kressig RW, Allali G, Beauchet O. Long-term practice of Jaques-Dalcroze eurhythmics prevents age-related increase of gait variability under a dual task. J Am Geriatr Soc 2005;53:728-729.

Marchant D, Sylvester JL, Earhart GM. Effects of a short duration, high dose contact improvisation dance workshop on Parkinson disease: a pilot study. Complement Ther Med 2010;18:184-190.

Moffet H, Noreau L, Parent E, Drolet M. Feasibility of an eight-week dancebased exercise program and its effects on locomotor ability of persons with functional class III rheumatoid arthritis. Arthritis Care Res 2000; 13:100-111.

Morgan C, Novak I, Badawi N. Enriched environments and motor outcomes in cerebral palsy: systematic review and meta-analysis. Pediatrics 2013;132:e735-746.

Morgan C, Novak I, Dale RC, Badawi N. Optimising motor learning in infants at high risk of cerebral palsy: a pilot study. BMC Pediatr 2015; 15:30. 
Shpigelman $\mathrm{CN}$, HaGani N. The impact of disability type and visibility on self-concept and body image: implications for mental health nursing. J Psychiatr Ment Health Nurs 2019;26:77-86.

Stribling K, Christy J. Creative dance practice improves postural control in a child with cerebral palsy. Pediatr Phys Ther 2017;29:365-369.

Taleporos G, McCabe MP. Body image and physical disability--personal perspectives. Soc Sci Med 2002;54:971-980.

Teixeira-Machado L, DeSantana JM. Effect of dance on lower-limb range of motion in young people with cerebral palsy: a blinded randomized controlled clinical trial. Adolesc Health Med Ther 2019;10:21-28.

Valvano J. Activity-focused motor interventions for children with neurological conditions. Phys Occup Ther Pediatr 2004;24:79-107.

Verschuren O, Wiart L, Hermans D, Ketelaar M. Identification of facilitators and barriers to physical activity in children and adolescents with cerebral palsy. J Pediatr 2012;161:488-494.

Yamaguchi T, Kadone H. Bodily expression support for creative dance education by grasping-type musical interface with embedded motion and grasp sensors. Sensors (Basel) 2017;17:1171. 